\title{
Superfluid-insulator transition in a periodically driven optical lattice
}

\author{
André Eckardt, Christoph Weiss, and Martin Holthaus \\ Institut für Physik, Carl von Ossietzky Universität, D-26111 Oldenburg, Germany
}

(Dated: October 23, 2005)

\begin{abstract}
We demonstrate that the transition from a superfluid to a Mott insulator in the Bose-Hubbard model can be induced by an oscillating force through an effective renormalization of the tunneling matrix element. The mechanism involves adiabatic following of Floquet states, and can be tested experimentally with Bose-Einstein condensates in periodically driven optical lattices. Its extension from small to very large systems yields nontrivial information on the condensate dynamics.
\end{abstract}

PACS numbers: 03.75.Lm, 03.75.Kk, 73.43.Nq

The Bose-Hubbard model plays an important role in condensed matter physics, since it embodies essential features of strongly interacting Bose systems in a minimal manner, namely the competition between kinetic and potential energy effects, and the resulting quantum phase transition from a superfluid to a Mott insulator [1, 2]. It describes Bose particles on a lattice with on-site interaction, so that particles occupying the same lattice site repel each other, while tunneling is allowed between adjacent sites. This is expressed by the Hamiltonian

$$
\hat{H}_{0}=-J \sum_{\langle i, j\rangle}\left(\hat{c}_{i}^{\dagger} \hat{c}_{j}+\hat{c}_{j}^{\dagger} \hat{c}_{i}\right)+\frac{U}{2} \sum_{j} \hat{n}_{j}\left(\hat{n}_{j}-1\right)
$$

where $\hat{c}_{j}^{(\dagger)}$ is an annihilation (creation) operator for a boson on the site labeled $j$, and $\hat{n}_{j}=\hat{c}_{j}^{\dagger} \hat{c}_{j}$ denotes the corresponding number operator. The first sum runs over all pairs of neighboring sites $i$ and $j$, with the matrix element $J$ quantifying the strength of the tunneling contact. Moreover, $U$ is the repulsion energy contributed by one pair of bosons located on the same site. Therefore, the characteristic dimensionless parameter is the ratio $U / J$ : When $U \ll J$, so that tunneling dominates, the ground state of the system describes a superfluid, whereas it has the properties of a Mott insulator when the interaction dominates, $U \gg J$. Mean-field theory [1, 2] gives the critical value $(U / J)_{c} \approx z \times 5.83$ for the transition in a lattice filled with one particle per site, which captures the case of a three-dimensional $(3 d)$ cubic lattice with coordination number $z=6$ reasonably well, whereas more refined methods [3] yield a Kosterlitz-Thouless transition with $(U / J)_{c} \approx 3.8$ for $d=1$.

After the model (11) had long been of primarily theoretical interest, it has found its laboratory realization with Bose-Einstein condensates in optical lattices [4, 5, 6]. In such systems, the expected transition has been observed upon varying the lattice depth, both for $d=3[7]$ and $d=1[8]$. In this Letter, we demonstrate that the transition from a superfluid to a Mott insulator can be induced in an altogether different manner which, in contrast to all scenarios studied before, hinges on the effect of a timedependent force, and which can be assessed experimentally with condensates in periodically modulated optical lattices. We will investigate a periodically forced BoseHubbard model for $d=1$, as described by the explicitly time-dependent Hamiltonian

$$
\hat{H}(t)=\hat{H}_{0}+K \cos (\omega t) \sum_{j} j \hat{n}_{j},
$$

where the equidistant sites are labeled according to their position in ascending order. The oscillating term, which mimics a monochromatic electric dipole potential with frequency $\omega$ and amplitude $K$, can be realized experimentally by periodically shifting the position of a mirror employed to generate the standing laser wave, and transforming to the co-moving frame of reference 9, , 10]. We will argue that the driven system (2) behaves, for sufficiently high frequencies, similar as the undriven system (11), but with the tunneling matrix element $J$ of the latter being replaced by the effective matrix element

$$
J_{\text {eff }}=J \mathrm{~J}_{0}(K /(\hbar \omega)),
$$

where $\mathrm{J}_{0}(x)$ denotes the ordinary Bessel function of order zero. Hence, the actual control parameter becomes $U / J_{\text {eff }}$, which can be varied by adjusting the parameters of the periodic modulation. This implies the possibility to switch between the superfluid and the insulator state by changing, e.g., the modulation strength $K$.

The rescaling (3) is not unfamiliar with periodically driven single-particle quantum systems. It occurs, among others, when a particle moves on a periodically forced $1 d$ lattice with nearest neighbor coupling [11, 12], such as an electron in a semiconductor superlattice 13]. It also underlies the $\mathrm{J}_{0}$-type renormalization of atomic $g$ factors in oscillating magnetic fields [14, 15], and the coherent destruction of tunneling of a particle in a periodically forced double-well [16, 17]. However, as will be discussed below, the many-body system (2) is significantly more involved when the thermodynamic limit is taken; the rescaling (3) then describes only part of the relevant physics.

Our analysis is based on quantum Floquet theory [18]: Since the Hamiltonian (2) depends periodically on time, $\hat{H}(t)=\hat{H}(t+T)$ with period $T=2 \pi / \omega$, there exists a complete set of solutions to the timedependent many-body Schrödinger equation of the form 
$\left|\psi_{n}(t)\right\rangle=\left|u_{n}(t)\right\rangle \exp \left(-\mathrm{i} \varepsilon_{n} t / \hbar\right)$, where the Floquet functions $\left|u_{n}(t)\right\rangle$ inherit the period of the driving force, satisfying $\left|u_{n}(t)\right\rangle=\left|u_{n}(t+T)\right\rangle$. Thus, Floquet states for periodically time-dependent quantum systems, obtained by solving the eigenvalue equation

$$
\left(\hat{H}(t)-\mathrm{i} \hbar \partial_{t}\right)\left|u_{n}(t)\right\rangle=\varepsilon_{n}\left|u_{n}(t)\right\rangle,
$$

constitute an analog of Bloch states known from spatially periodic crystals; the eigenvalues $\varepsilon_{n}$, which describe the time evolution of these states in close analogy to the evolution of energy eigenstates, are called quasienergies. While in solid-state physics quasimomenta are defined up to an integer multiple of a reciprocal lattice vector, quasienergies are defined up to an integer multiple of $\hbar \omega$ : If $\left|u_{n}(t)\right\rangle$ solves Eq. (4) with eigenvalue $\varepsilon_{n}$, and $m=0, \pm 1, \pm 2, \ldots$, then $\left|u_{n}(t)\right\rangle \exp (\mathrm{i} m \omega t)$ is a $T$ periodic eigensolution with quasienergy $\varepsilon_{n}+m \hbar \omega$. The quasienergy spectrum of a periodically time-dependent quantum system thus possesses a Brillouin zone-like structure, the width of one zone being $\hbar \omega$.

We then employ the Floquet basis

$$
\left|\left\{n_{j}\right\}, m\right\rangle=\left|\left\{n_{j}\right\}\right\rangle \exp \left[-\mathrm{i} \frac{K}{\hbar \omega} \sin (\omega t) \sum_{j} j n_{j}+\mathrm{i} m \omega t\right],
$$

where $\left|\left\{n_{j}\right\}\right\rangle$ indicates a Fock state with $n_{j}$ particles on the $j$ th site, and $m$ again accounts for the zone structure. The eigenvalue problem (4) refers to an extended Hilbert space of $T$-periodic functions, in which the time variable is regarded as a coordinate [19], so that the scalar product in that space is given by

$$
\langle\langle\cdot \mid \cdot\rangle\rangle=\frac{1}{T} \int_{0}^{T} \mathrm{~d} t\langle\cdot \mid \cdot\rangle,
$$

i.e., by the usual scalar product $\langle\cdot \mid \cdot\rangle$ combined with timeaveraging. Hence, the quasienergies are obtained by computing the matrix elements of the operator $\hat{H}(t)-\mathrm{i} \hbar \partial_{t}$ in the basis (5) with respect to the scalar product (6), and diagonalizing. Denoting the Hamiltonian $\hat{H}_{0}$ with $J=0$, which is diagonal in the basis (5), by $\hat{H}_{\text {int }}$, and its $J$-proportional tunneling term by $\hat{H}_{\text {tun }}$, we find

$$
\begin{aligned}
& \left\langle\left\langle\left\{n_{j}^{\prime}\right\}, m^{\prime}\left|\hat{H}(t)-\mathrm{i} \hbar \partial_{t}\right|\left\{n_{j}\right\}, m\right\rangle\right\rangle \\
= & \delta_{m^{\prime}, m}\left[\left\langle\left\{n_{j}^{\prime}\right\}\left|\hat{H}_{\mathrm{int}}\right|\left\{n_{j}\right\}\right\rangle+m \hbar \omega\right] \\
+ & s^{m^{\prime}-m} \mathrm{~J}_{m^{\prime}-m}(K /(\hbar \omega))\left\langle\left\{n_{j}^{\prime}\right\}\left|\hat{H}_{\mathrm{tun}}\right|\left\{n_{j}\right\}\right\rangle,
\end{aligned}
$$

where $s=\sum_{j}\left(n_{j}^{\prime}-n_{j}\right) j= \pm 1$, since $\hat{H}_{\text {tun }}$ only transfers one particle by one site. We observe that with respect to the "photon" index $m$ this matrix has a transparent block structure: The diagonal blocks with $m=m^{\prime}$ reproduce the matrix which yields the eigenvalues of the undriven system, but with $J$ replaced by $J_{\text {eff }}$ according to Eq. (3), and replicas shifted by integer multiples of $\hbar \omega$. These

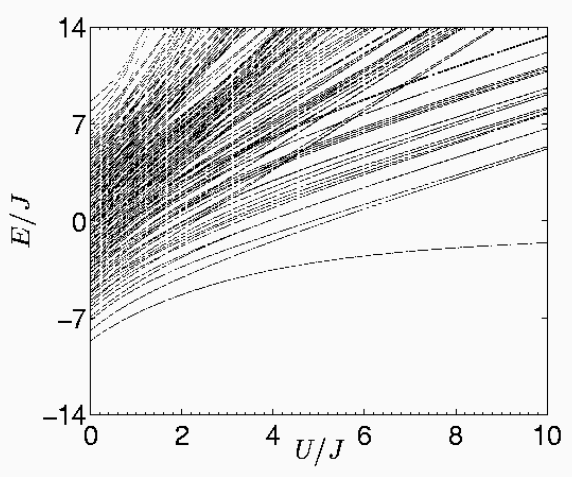

FIG. 1: Exact energy spectrum of a small one-dimensional undriven lattice (1) with $M=5$ sites and $N=5$ particles, versus Mott-Hubbard parameter $U / J$. The splitting-off of the ground state with increasing interaction strength can be regarded as a precursor of the quantum phase transition.

blocks are coupled by nondiagonal ones proportional to Bessel functions $\mathrm{J}_{m^{\prime}-m}(K /(\hbar \omega))$. Obviously, the anticipated rescaling (3) holds only to the extent that these couplings can be neglected. This will be the case, at least in a perturbative sense, if the block separation $\hbar \omega$ is much larger than both the energy scale $J$ of the coupling and the energy scale $U$ associated with the diagonal blocks, i.e., for high frequencies $\hbar \omega \gg \max \{J, U\}$.

To demonstrate that this reasoning is justified, we present numerical results for small systems. Figure 1 depicts the exact energy spectrum for a one-dimensional undriven model (1) with $N=5$ particles on $M=5$ sites. Even here, the precursor of the superfluid-insulator transition already is apparent: With increasing $U / J$ the system's ground state, associated with a uniform distribution of the particles over the sites, splits off from the group of excited states, which describe various patterns of particle-hole excitations. In the limit of an infinitely large system, $N \rightarrow \infty$ and $M \rightarrow \infty$ with $N / M=1$ held constant, the excited states form continuous energy bands; the ground state then splits off from the lowest band at a finite $(U / J)_{c}[\underline{3}]$. This separation of an individual state from the continuum indicates the transition to the Mott insulator state.

For comparison, Fig. 2] shows the first Brillouin zone of numerically computed quasienergies for the driven system (21), again with $N=M=5$, scaled frequency $\hbar \omega / J=14$, and scaled driving amplitude $K / \hbar \omega=1.5$. Since $\mathrm{J}_{0}(1.5) \approx 0.5$, this set of parameters allows for a convenient test of the hypothesis (3): With $J_{\text {eff }} \approx J / 2$, the quasienergy spectrum of the driven system (2) for a given parameter $U / J$ should correspond (apart from its zone structure) to the energy spectrum of the undriven system (11) with the same $U / J_{\text {eff }}$, which is about $2 U / J$. This is borne out, to remarkable accuracy, by 


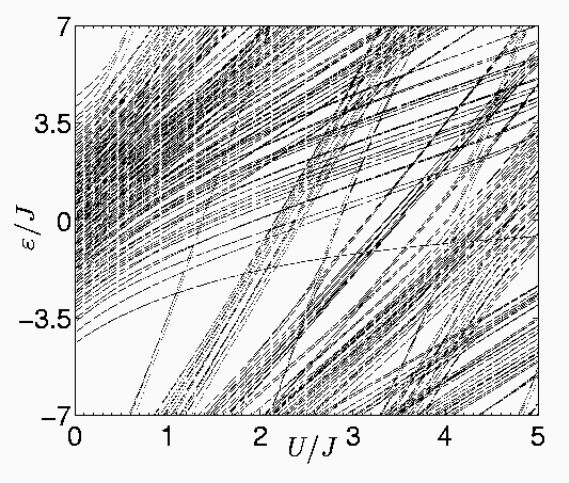

FIG. 2: First Brillouin zone of the exact quasienergy spectrum of the driven $1 d$ system (2) with $N=M=5, \hbar \omega / J=14$, and $K / \hbar \omega=1.5$. This spectrum is recovered approximately from the energy eigenvalues shown in Fig. 1 if $J$ is replaced by $J_{\text {eff }} \approx J / 2$ here, and the energies are taken modulo $\hbar \omega$.

a comparison of Figs. 1 and 2 With the scales of the respective axes differing by a factor of two, the eigenvalues plotted in Fig. 2 almost equal those in Fig. 1. As a consequence of the Brillouin zone structure, quasienergy eigenvalues which disappear at the upper zone boundary reappear again at the lower one. This reappearance is a source of substantial complications: States originating from different Brillouin zones are coupled through the matrix elements neglected in the explanation of the renormalization (3), so that many apparent level crossings in Fig. 2 actually are tiny avoided crossings. It is the high-frequency condition $\hbar \omega \gg \max \{J, U\}$ which guarantees that these avoided crossings remain too narrow to be resolved. If this condition is not met, a multitude of large avoided crossings appears in the spectrum, thus revealing typical signatures of quantum chaos [20].

Figure 3 shows quasienergies for $U / J=3 \mathrm{kept}$ fixed, again for $\hbar \omega / J=14$, as functions of the scaled amplitude $K /(\hbar \omega)$. For $K /(\hbar \omega) \approx 2.4$, close to the first zero of $\mathrm{J}_{0}$, the tunneling contact is quenched almost entirely, so that the various bands of particle-hole excitations collapse. In the vicinity of this point, the Floquet state evolving from the unperturbed ground state must have the properties of a Mott insulator, although the ground state of the undriven system describes a superfluid for $U / J=3$.

To verify this conclusion, we have solved the timedependent Schrödinger equation for a system with $N=$ $M=7$ and $U / J=3$, initially prepared in its superfluid ground state and then subjected to periodic forcing with frequency $\hbar \omega / J=14$, and an amplitude $K /(\hbar \omega)$ which increases linearly from 0 to 2.4 during the first $100 \mathrm{cy}-$ cles $T$, then stays constant for another 100 cycles, and finally is linearly ramped down to zero between $t=200 \mathrm{~T}$ and $t=300 T$. Experimentally, the superfluid phase is detected by a sharply peaked reciprocal lattice pattern in

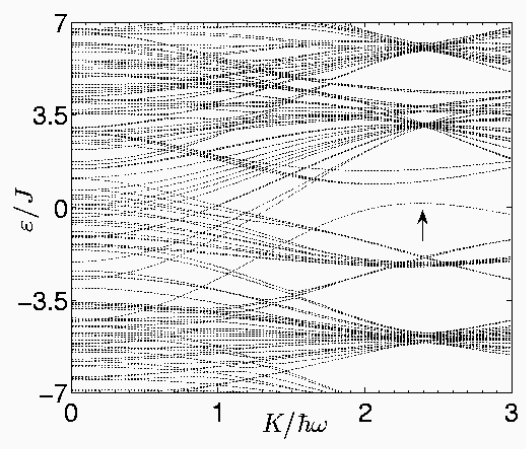

FIG. 3: First Brillouin zone of the exact quasienergy spectrum of the driven $1 d$ system with $N=M=5, \hbar \omega / J=14$, and $U / J=3$, versus scaled driving amplitude $K / \hbar \omega$. For $K / \hbar \omega \approx 2.4$, close to the first zero of $\mathrm{J}_{0}$, the tunneling contact is (almost) switched off, resulting in a collapse of the different bands. In the vicinity of this value, the Floquet state evolving from the system's ground state (marked by the arrow) has the properties of a Mott insulator, even though the undriven system's ground state is superfluid for $U / J=3$.

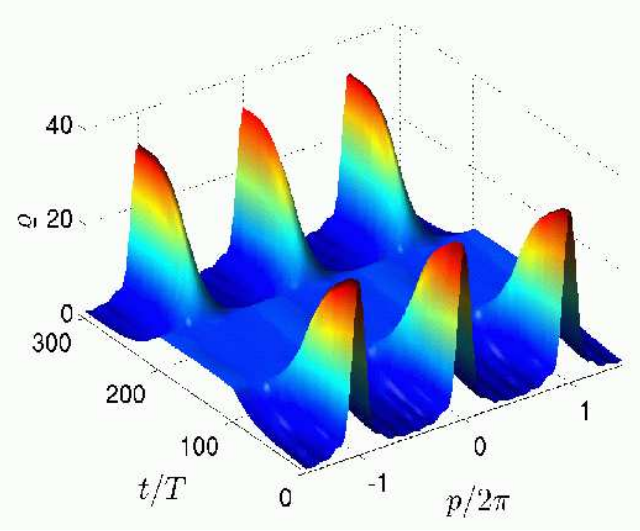

FIG. 4: Time evolution of the momentum distribution $\varrho(p)$ obtained by solving the time-dependent Schrödinger equation for $N=M=7, \hbar \omega / J=14$, and a slowly varying amplitude $K /(\hbar \omega)$ which is linearly ramped up to 2.4 during the first 100 cycles $T$, then stays constant for another $100 T$, and is ramped down to zero during the final 100 cycles. The system was initially in its ground state; the distribution was recorded at integer multiples of $T$. The disappearance and reappearance of the peak pattern signals the transition from the superfluid to the Mott state and back.

the momentum distribution, $\varrho(p)=\sum_{j, k}\left\langle c_{j}^{\dagger} c_{k}\right\rangle \mathrm{e}^{-\mathrm{i} p(j-k)}$, which can be measured by time-of-flight absorption imaging. (Here, $p$ is given in multiples of $\hbar /$ lattice constant.) Figure 4 depicts our result: Since Floquet states respect an approximate adiabatic principle 21], the initial superfluid ground state is first adiabatically transformed into a Mott insulator state, as witnessed by the disappearance 
of the peaked momentum distribution, and then transformed back to the inital state, apart from a remaining excitation of other states totalling to a few percent. This is a major result: The amplitude of the periodic force decides whether the system is superfluid, or in a Mott insulator state.

When extrapolating from these model calculations to large systems, two issues have to be considered. Firstly, when increasing the number of lattices sites while maintaining an occupancy of one particle per site, say, the quasienergy levels fill the Brillouin zone densely; in the thermodynamic limit, the spectrum probably is a continuum. Then there will be no "sharp" Floquet state evolving from the ground state, but rather a resonance with a finite lifetime, due to the residual couplings to other states. Starting from an undriven, infinite system with a superfluid ground state, and switching on the periodic force, we conjecture that a Mott-insulator-like resonance appears at that amplitude $K$ which, after rescaling according to Eq. (3), corresponds to that tunneling matrix element $J$ which marks the quantum phase transition in the undriven system. Secondly, for an infinitely large lattice there is no adiabatic limit when switching on the driving force [22]; turn-on and turn-off necessarily have to take place within a short interval. Our calculations indicate that adiabatic following can even be improved by shortening the turn-on time, since Landau-Zener transitions at narrow avoided crossings, possibly corresponding to condensate heating, then are suppressed.

While these issues are still not covered by rigorous mathematical theorems on periodically driven quantum systems [23], and remain out of reach of even most powerful supercomputers, they can be addressed in the laboratory. In experiments with cold atoms in driven optical lattices [10], narrowing of Bloch bands compatible with the rescaling (3) has already been observed, even though the single-band regime has not been reached. Employing Bose-Einstein condensates in optical lattices in order to realize the driven Bose-Hubbard model (2), one has to respect not only the high-frequency condition $\hbar \omega>\max \{J, U\}$ required for the approximate Besselfunction rescaling (3), but there is the obvious additional condition $\hbar \omega<\Delta$, where $\Delta$ denotes the gap between the lowest two Bloch bands of the undriven lattice, in order to exclude transitions to higher band states. Elementary estimates in the spirit of Ref. [5] suggest that under typical conditions (as provided by ${ }^{87} \mathrm{Rb}$ atoms in a lattice created by laser radiation of $\lambda=852 \mathrm{~nm}$ wavelength [7]) this leaves a viable window of frequencies in the low $\mathrm{kHz}$ regime. For higher filling factors, or in $3 d$ lattices with forcing in all three directions, the critical parameter $(U / J)_{c}$ becomes much larger, allowing one to employ deeper lattices with larger band gap $\Delta$, and hence to work with still higher frequencies without violating the single-band approximation. A quantity of key interest in such experiments will be the extent to which, after start- ing from a superfluid ground state, then ramping up the force into the insulator regime and ramping it down again as in Fig. 4 the superfluid peak pattern reappears, providing information on both the lifetime of the conjectured Mott-like resonance state and the degree of adiabatic following, or, more generally, on the extent to which the quantum evolution of a mesoscopic matter wave can be guided even under critical conditions. Thus, the scenario envisioned here is not intended as a look at the common superfluid-insulator transition from a different angle, but aims at obtaining genuinely new, nontrivial information on condensate dynamics.

This work was supported by the DFG through the Priority Programme SPP 1116. A.E. acknowledges a fellowship from the Studienstiftung des deutschen Volkes.

[1] M. P. A. Fisher, P. B. Weichman, G. Grinstein, and D. S. Fisher, Phys. Rev. B 40, 546 (1989).

[2] S. Sachdev, Quantum Phase Transitions (Cambridge University Press, Cambridge, 1999).

[3] N. Elstner and H. Monien, Phys. Rev. B 59, 12184 (1999).

[4] D. Jaksch, C. Bruder, J. I. Cirac, C. W. Gardiner, and P. Zoller, Phys. Rev. Lett. 81, 3108 (1998).

[5] W. Zwerger, J. Opt. B: Quantum Semiclass. Opt 5, S9 (2003).

[6] D. Jaksch and P. Zoller, Ann. Phys. (N.Y.) 315, 52 (2005).

[7] M. Greiner, O. Mandel, T. Esslinger, T. W. Hänsch, and I. Bloch, Nature 415, 39 (2002).

[8] T. Stöferle, H. Moritz, C. Schori, M. Köhl, and T. Esslinger, Phys. Rev. Lett. 92, 130403 (2004).

[9] R. Graham, M. Schlautmann, and P. Zoller, Phys. Rev. A 45, R19 (1992).

[10] K. W. Madison, M. C. Fischer, R. B. Diener, Q. Niu, and M. G. Raizen, Phys. Rev. Lett. 81, 5093 (1998).

[11] D. H. Dunlap and V. M. Kenkre, Phys. Rev. B 34, 3625 (1986).

[12] M. Holthaus, Phys. Rev. Lett. 69, 351 (1992).

[13] T. Meier, G. von Plessen, P. Thomas, and S. W. Koch, Phys. Rev. B 51, 14490 (1995).

[14] S. Haroche, C. Cohen-Tannoudji, C. Audoin, and J. P. Schermann, Phys. Rev. Lett. 24, 861 (1970).

[15] C. Cohen-Tannoudji, Atoms in Electromagnetic Fields (World Scientific, Singapore, 1994).

[16] F. Grossmann, P. Jung, T. Dittrich, and P. Hänggi, Z. Phys. B 84, 315 (1991).

[17] M. Grifoni and P. Hänggi, Phys. Rep. 304, 229 (1998).

[18] J. H. Shirley, Phys. Rev. 138, B979 (1965).

[19] H. Sambe, Phys. Rev. A 7, 2203 (1973).

[20] F. Haake, Quantum Signatures of Chaos (Springer Series in Synergetics 54, Berlin, 2004).

[21] H. P. Breuer and M. Holthaus, Z. Phys. D 11, 1 (1989).

[22] D. W. Hone, R. Ketzmerick, and W. Kohn, Phys. Rev. A 56, 4045 (1997).

[23] J. S. Howland, Quantum Stability. In: Spinger Lecture Notes in Physics 403, 100 (1992). 